\title{
LA MITIGAZIONE NEL TESTO MEDICO-SCIENTIFICO
}

\author{
MITIGATION IN BIOMEDICAL SCIENTIFIC TEXTS
}

\section{LA MITIGACIÓN EN EL TEXTO CIENTÍFICO-MÉDICO}

\author{
Silvia IERARDi \\ Gruppo di ricerca di Genova, University of Genova ${ }^{1}$ \\ silviaierardi@yahoo.it \\ Daniele Stancampiano \\ Gruppo di ricerca di Genova, University of Genova ${ }^{2}$ \\ d.danielestancampiano@gmail.com
}

Recibido: 01/03/2017

Aceptado: 09/06/2017

\section{Sommario}

In questo contributo, ci proponiamo di avanzare alcune proposte per un'analisi pragmatica della mitigazione nel testo medico-scientifico. Abbiamo cercato di riadattare il modello della mitigazione di Caffi (2001) al testo scritto medico-scientifico, analizzando alcuni articoli del British Medical Journal. Proponiamo una

\section{Resumen}

En esta contribución, proponemos avanzar algunas propuestas para un análisis pragmático de la mitigación en el texto médico-científico. Hemos intentado reajustar el modelo de mitigación Caffi (2001) al texto médicocientífico escrito, analizando algunos artículos del British Medical Journal. Proponemos una

1 Prin 2012 CUP D38C 13000610001 gruppo di ricerca di Genova. Ricerca inserita nell'ambito del progetto di ricerca di interesse nazionale PRIN 2012, codice CUP D38C 130006 10001, intitolato Certezza e incertezza nella comunicazione scientifica biomedica. Coordinatore scientifico nazionale: Andrzej Zuckowski, Università di Macerata; coordinatore scientifico dell'unità di ricerca di Genova: Claudia Caffi, Università degli Studi di Genova, i cui primi risultati sono stati discussi in un Workshop tenutosi all'Università di Genova nell'aprile 2016 (Caffi et alii 2016; Mey, in press).

2 Prin 2012 CUP D38C 13000610001 gruppo di ricerca di Genova. Ricerca inserita nell'ambito del progetto di ricerca di interesse nazionale PRIN 2012, codice CUP D38C 130006 10001, intitolato Certezza e incertezza nella comunicazione scientifica biomedica. Coordinatore scientifico nazionale: Andrzej Zuckowski, Università di Macerata; coordinatore scientifico dell'unità di ricerca di Genova: Claudia Caffi, Università degli Studi di Genova, i cui primi risultati sono stati discussi in un Workshop tenutosi all'Università di Genova nell'aprile 2016 (Caffi et alii 2016; Mey, in press).

Para citar este artículo / To cite this article: Ierardi, Silvia y Stancampiano, Daniele (2018): La mitigazione nel testo medico-scientifico. García Ramón, Amparo y Soler Bonafont, María Amparo (Eds.): ELUA: Estudios de atenuación en el discurso, Anexo IV, págs. 179-196.

Enlace / Link: http://dx.doi.org/10.14198/ELUA2018.Anexo4.10 
classificazione di due tipi di mitigatori, i bushes (cespugli) e gli hedges (siepi), e alcune riflessioni circa il rapporto tra bushes e incertezza e tra hedges ed evidenzialità.

PAROLE CHIAVE: mitigazione, testo scientifico, hedges, evidenzialità, vaghezza. clasificación de dos tipos de mitigadores, bushes y hedges, y algunas reflexiones sobre la relación entre los arbustos y la incertidumbre y entre las coberturas y la evidencia.

PALABRAS CLAVE: mitigación, texto científico, mitigadores, evidencia, vaguedad.

\begin{abstract}
In this paper, we propose to make some categorizations for pragmatic analysis of mitigation in the medical-scientific text. We tried to apply the pattern of mitigation presented by Caffi (2001) to medical-scientific text, by analyzing some articles in the British Medical Journal. We propose a classification of two types of strategies, bushes (cespugli) and hedges (siepi), and some thoughts about the relationship between bushes and vagueness and between hedges and evidentiality.

KEY WORDS: mitigation, scientific text, hedges, evidentiality, vagueness.
\end{abstract}

\title{
1. MITIGAZIONE E TESTO SCIENTIFICO: SPUNTI DI ANALISI
}

\subsection{Il testo scientifico}

Da oltre trent'anni, un gran numero di studi multidisciplinari, dalla sociologia alla pragmatica linguistica, passando per la retorica e l'epistemologia, si sono concentrati sull'analisi del testo scientifico (Polany 1946, 1962; Merton 1968, 1981; Latour e Woolgar 1979; Van Eemeren e Grootendorst 1982; Parret 1984; Gross 1985; Bazerman 1988, 1991; Swales 1990; Mangani 1991; Halliday e Martin 1993; Grabe e Kaplan 1997; Meyer 1997; Skelton 1997; Latour 1998, 2000; Hyland 1998, 2002, 2009; Knorr-Cetina 1999, 2007; Bastide 2001; Halliday 2004a, 2004b; Gallardo 2005; Hyland e Guinda Sancho 2012; Feyerabend 2013). Metaforicamente, si potrebbero riassumere i risultati di queste analisi affermando che le coordinate deittiche delle attività degli scienziati convergono tutte verso una unica origo (Bühler 1983), che è il laboratorio.

Questo luogo acquista il significato di totem (Lévi-Strauss 1964) dell'attività scientifica: infatti è all'interno del laboratorio che lo scienziato lavora, ricerca, setacciando la realtà fisica indifferenziata e cercando di portare alla luce l'elemento supposto o ipotizzato e, nel farlo, adotta strumenti che lo aiutino a tendere verso la massima oggettività ed empiricità possibili. Questo procedimento segue un metodo che vigila sopra ogni fase e serve ad omogeneizzare processi e risultati, facilitando il confronto e lo scambio di dati fra laboratori ed assurgendo, a sua volta, come garante unico per la scienza in generale. Proprio come i totem descritti da Lévi-Strauss (Ib.), si insatura un processo dialogico molto stretto tra la definizione di scienza ed il fare scienza, tanto che i due momenti arrivano ad identificarsi 
l'uno con l'altro. Lo scienziato, che agisce in laboratorio applicando le procedure vincolanti del metodo scientifico, viene a sua volta agito dal metodo stesso, che si impone come unico veicolo per poter chiamare quell'agire indifferenziato agire scientifico; al contrario, il metodo scientifico, che assurge a garante assoluto dell'attività del laboratorio, nasce e si perfeziona con la prassi della ricerca, in un meccanismo epistemico che è proprio dei paradigmi a posteriori, a vocazione fortemente empirica.

Il laboratorio funge anche da conclave della scienza dove sono ammessi solo gli addetti ai lavori ed extra omnes. Per mantenere i vincoli di chiarezza e replicabilità dell'esperimento, essenziali a scacciare il demone della manipolazione dei dati e scoraggiare il plagio, l'attività del laboratorio si è dotata di una voce (Bazerman 1988, 1981), un proprio resoconto, che per la pretesa di fedeltà percettiva assoluta nel riportare ogni singola fase dell'esperimento può essere definito usando il termine di faccia, immagine (Goffman 1971, 1987): tale prodotto è l'Articolo di Ricerca scientifico $(R A s)$. La faccia è la prima cosa che si vede, il biglietto da visita; anche per la scienza, il $R A s$ è la presentazione più immediata e più usufruita del lavoro nei laboratori e per questo deve veicolarne non solo i contenuti, ma soprattutto il metodo. Precisione, sovrabbondanza di dettagli, chiarezza espositiva, impostazione empirica e massima oggettività non sono solo semplici parametri di un genere testuale, ma trasposizioni attraverso le quali un genere epistemico, un modo di strutturare e sistematizzare la conoscenza quale è il paradigma scientifico, diviene genere testuale. E mentre agisce sul $R A s$, permettendo che questo diventi il maggiore veicolo della propria immagine distintiva, il paradigma conoscitivo ne viene a sua volta agito, strutturando il proprio fare scienza anche guardando alla sua comunicabilità.

\subsection{La mitigazione}

L'idea generale di mitigazione, così come intesa e più volte esplicitata da Caffi (1994, 2001), è connessa a doppio filo con quella di consapevolezza metapragmatica (Caffi 1994, 2001, in pubblicazione), capacità del singolo individuo di usare le proprie competenze per ottimizzare la propria presenza nel mondo, rendendo le proprie scelte linguistiche le più adeguate possibili. Citando Caffi (in pubblicazione):

Il concetto di appropriatezza va negoziato tra gli interlocutori. L'appropriatezza di una scelta è giudicata in base a criteri linguistici (è il nostro sapere sulla lingua), enciclopedici (è il nostro sapere sul mondo, che comprende l'esperienza di quella data situazione, o "frame") e psicologici (è il nostro sapere su noi stessi, e sugli altri). Il sapere multiplo che si manifesta nella mitigazione investe zone procedurali della consapevolezza metapragmatica.

Il modello della mitigazione (Caffi 2001) è olistico ed integrato: olistico in quanto viene dedotto dai dati empirici raccolti in ogni particolare ambito di studi, rendendolo allo stesso tempo flessibile e adattabile; integrato poiché la mitigazione agisce trasversalmente attraverso vari ambiti disciplinari, quali la retorica e la stilistica. Nel mettere in pratica le strategie mitiganti, ogni parlante, più o meno consapevole, sa di poter agire su più livelli, a diverse intensità di intervento, dando vita a fenomeni di co-varianza su più dimensioni (o parametri), convergenti (ad esempio il rafforzamento del parametro della precisione per aumentare 
il parametro della certezza) oppure divergenti. Caffi (2001, 2007, in pubblicazione), a tal proposito, riprende spesso la metafora di Spitzer (2007): "come in musica l'uso del pedale".

Che si parli di culture epistemiche (Knorr-Cetina 1999, 2007), società dei rischi (Beck 2010), società liquida (Bauman 2003) oppure società dell'informazione (Castellas 2012), rimane indubbio che il paradigma epistemico della scienza sia dominante oggi, tanto da permeare le abitudini della vita quotidiana. Qui risiede il motivo della nostra ricerca, costituita da un'analisi qualitativa dei fenomeni di mitigazione all'interno degli articoli del British Medical Journal:

1. ad intra, l'obiettivo è vincere la sfida di fondo del modello Caffi (2001), credendo che il sistema di conoscenza predominante oggi non possa esimersi dall'uso della mitigazione, vista appunto come ottimizzazione del nostro saper stare al mondo. Occorrerà sicuramente riadattare il modello della mitigazione, nato dall'analisi di dati empirici esclusivamente conversazionali, allo studio di un genere esclusivamente testuale e altamente definito e strutturato sul livello stilistico e retorico. Bisognerà inoltre testare l'elasticità delle categorie proposte dal modello e le loro prototipicità, andando a sviscerarne gli usi impliciti ed espliciti all'interno del genere testuale oggetto delle nostre analisi;

2. ad extra, questo primo approccio apre ad una prospettiva di studio inesplorata quanto necessaria oggi, che porta dal semantico al pragmatico, dall'illocutorio al perlocutorio; una prospettiva che speriamo non si fermi ai generi testuali definibili e definiti, ma che si imponga di indagare, attraverso e grazie alla trasversalità della mitigazione, il funzionamento del nostro modo di ricevere, organizzare, gerarchizzare e condividere la conoscenza. Scienza tout court, divulgazione scientifica, letteratura giuridica, messaggi e dibattiti politici, omelie papali, recensioni sul web, sono tutte fonti di informazione di cui usufruiamo quotidianamente e che usiamo con precisi obiettivi (Sbisà 1978, 2001, 2009) perlocutori e che a volte producono conseguenze (Ead 2001.) perlocutorie inaspettate. Se mitigazione può significare, all'interno della cornice di competenza metapragmatica (Caffi 1994, 2001, in pubblicazione), ottimizzazione dell'interazione, vista come insieme integrato di metodi e scopi, potrebbe esserci allora un legame, ancora da analizzare, fra mitigazione e permeabilità del messaggio, che accrescerebbe ulteriormente il valore d'interesse di ulteriori studi in tal senso.

\subsection{Il corpus}

Il corpus da noi analizzato è costituito da quaranta articoli della sezione Research del British Medical Journal, disponibili in modalità open access sul sito della rivista. I testi appartengono alle annate 2013 e 2015 e sono stati selezionati in modalità random.

Dall'analisi degli articoli selezionati è emerso come essi siano di due tipologie, che definiamo research e review (o meta-analisi). I primi presentano i risultati di ricerche in corso o appena concluse; i secondi sono rassegne di studi precedentemente pubblicati su una determinata tematica medico-scientifica. Gli articoli di research e quelli di review presentano caratteristiche molto differenti e un uso e una frequenza diversi dei markers di mitigazione. In questo contributo presenteremo i risultati ottenuti con l'analisi degli articoli di research. 


\section{BUSHES NEL BRITISH MEDICAL JOURNAL}

Tra le strategie di mitigazione individuate nel corpus del British Medical Journal da noi analizzato, vi sono diverse ricorrenze di bushes (Caffi 2001). I bushes corrispondono a quei mezzi linguistici chiamati da G. Lakoff (1973 [1972]), hedges, e da Prince et alii (1982), approximators; agiscono sull'ambito proposizionale dell'enunciato, rendendo vago il referente della proposizione. Con riferimento alle classificazioni di mezzi che veicolano vaghezza più utilizzate dalla letteratura, in particolare quella di Channell (1994), ripresa da Overstreet (2011), proponiamo la seguente classificazione dei bushes, che procede in base al tipo di referente approssimato (cfr. Figura 1):

1. Referente numerico (rounders)

1. Approssimatori di quantità numeriche

1. Numerali

2. Approssimatori di quantità

3. General extenders

2. Approssimatori di tempo

2. Referente non numerico

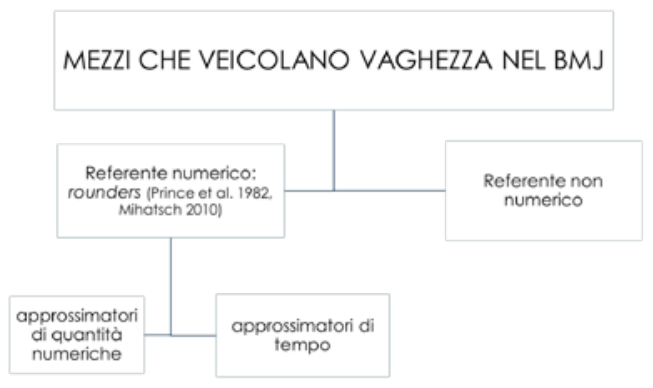

Figura 1. Mezzi che veicolano vaghezza nel BMJ.

Una distinzione analoga, tra mezzi che veicolano vaghezza agendo su termini il cui referente è numerico e mezzi che veicolano vaghezza agendo su termini il cui referente non è numerico, è già stata proposta da Carter e McCarthy (2006), il cui lavoro è basato su Channell (1994). Questi distinguono tra approximators, definiti come all forms relating to numbers or quantities, e vague language, cioè nouns with vague reference (things), downtoners (sort of), general extenders (or something) (Overstreet 2011:, p. 298). Il nostro modello, costruito sul e per il testo medico-scientifico, si differenzia in alcuni punti e precisa alcune categorie di approssimatori.

\subsection{Rounders}

I rounders, già individuati nel testo medico parlato da Prince et alii (1982), sono approssimatori che intervengono su una quantità numerica rendendola vaga o sono loro stessi termini con un referente numerico vago. Nel nostro corpus abbiamo individuato 473 rounders: sono i bushes che ricorrono più frequentemente. 
Procedendo nel classificare i bushes seguendo il criterio del tipo di referente approssimato, li abbiamo suddivisi in: approssimatori di quantità numeriche e approssimatori di quantità non strettamente numeriche.

I primi, gli approssimatori di quantità numeriche, rappresentano 1'86\% dei rounders del corpus. Possono veicolare vaghezza mediante l'uso dei numerali, di approssimatori di quantità o di general extenders.

I numerali sono approssimatori quando vengono utilizzati in luogo dell'indicazione della quantità precisa, quindi quando sono numeri tondi (Channell 1994, Bazzanella 2011, Overstreet 2011) (cfr. esempio (1)) o quando indicano intervalli di valori (cfr. esempio (2)) o medie matematiche.

Gli approssimatori di quantità sono mezzi che veicolano vaghezza modificando una quantità precisa. Corrispondono a quelli che nella classificazione di Channell (1994) sono definiti approximators (e.g. about...) e a quelli che nella classificazione di Overstreet (2011) sono definiti vague amounts (e.g. several) (cfr. esempio (1)). Nella nostra classificazione, queste categorie ricadono sotto la stessa etichetta di approssimatori di quantità in quanto entrambe le tipologie di mezzi veicolano vaghezza, hanno referenti analoghi e occupano la medesima posizione nell'ordine sintattico della frase: modificano sempre una quantità numerica che precedono.

Infine, i general extenders (referring vaguely to category, Channell 1994, Adolphs et al. 2007, Overstreet 2011, Voghera 2012) estendono la quantità espressa in maniera indefinita, quindi vaga. Spesso, la quantità estesa è indicata mediante un numero tondo; gli autori ricorrono quindi all'utilizzo di clusters di markers, cioè markers che operano in combinazione tra loro, caratterizzati dalla struttura: numerale + general extender (cfr. esempio (1)).

(1) If the societal willingness to pay per QALY was $£ 10000$ or higher, the probabilities that hybrid prostheses were the most cost effective prosthesis type were about $75 \%$ for men and $70 \%$ for women. The corresponding probabilities for cementless prostheses were about $25 \%$ for men and $30 \%$ for women, and around $1 \%$ for cemented prostheses. For men aged 60 or 80 and for women aged 60, hybrid prostheses gave the highest expected net benefit and had the highest probability of being the most cost effective prosthesis type (Figura 2 and Table 4) (British Medical Journal, Feb 2013, p. 5).

(2) The risk of suicide is between $6 \%$ and $10 \%, 10$ times higher than in the non-psychiatric population (British Medical Journal, Jun 2013: 1).

L'esempio (1) presenta diversi tipi di rounders: tutti i numerali sono approssimatori, in quanto numeri tondi o, mediante un modificatore, indicano un intervallo (aged 60); or higher è un general extender; about, in tutte le sue ricorrenze, è un approssimatore di quantità. Inoltre, in quest'esempio, sono presenti diversi clusters di marker: il numero tondo 10000 è seguito dal general extender "or higher" e le percentuali tonde 75 e 70 sono precedute dall'approssimatore about. La combinazione di più markers è una caratteristica tipica dei mitigatori presenti anche in altri contesti (Caffi 2001).

L'esempio (2) presenta un altro caso, dopo quello analizzato sopra, di rounder costituito da intervallo numerico: $6 \%$ e $10 \%$, numerali precisi, sono approssimati dal modificatore between [...] and che li rende un intervallo. 
La seconda categoria di rounders, gli approssimatori di quantità non strettamente numeriche, è principalmente costituita da indicazioni temporali non precise, ottenute mediante formule, quali: Over the past few years, In recent decades e recent outbreaks (rispettivamente negli esempi (3), (4) e (5)). Chiamiamo questa categoria di rounders approssimatori di tempo o determinazioni vaghe di tempo.

(3) Over the past few years, several new treatments have been introduced, most notably the new class of oral "incretin" drugs known as the dipeptidyl peptidase-4 (DPP-4) inhibitors (British Medical Journal, Apr 2013: 1).

(4) In recent decades, concern has mounted regarding the high prevalence and costs associated with cardiovascular disease, with growing interest in altering risk factors and reversing this global epidemic (British Medical Journal, Jan 2013: 1).

(5) Reasons for the increase are likely varied, but studies of recent outbreaks have found that acellular pertussis vaccines for children are less effective than earlier whole cell formulations $^{1}$, and that protection wanes substantially after the last dose ${ }^{2}$.

\subsection{Bushes con referente non numerico}

Questo tipo di bushes si differenzia dai rounders in quanto non agisce su un referente numerico. Abbiamo individuato 350 bushes con referente non numerico. La maggior parte di essi presenta la struttura: articolo indeterminativo + referente non numerico (sintagma nominale). L'articolo indeterminativo, per sua stessa natura, non determina in maniera precisa il sintagma nominale, rendendolo in tal modo indefinito, vago (esempio (6)).

Meno frequenti, ma tuttavia presenti, sono altre indicazioni vaghe del referente, in cui il referente non numerico è parte di un sintagma nominale o verbale complesso, costituito da nome o verbo - cioè il referente non numerico - e modificatore (del nome o del verbo). Quest'ultimo è costituito da un aggettivo o da un avverbio, che presentano vaghezza a livello semantico (De Mauro 1982, Ballmer e Pinkal 1983, Voghera 1997) o che, comunque, non intervengono sul referente con un'azione di specificazione (esempi (5), che riporto nuovamente per comodità di lettura, e (6)).

(5) Reasons for the increase are likely varied, but studies of recent outbreaks have found that acellular pertussis vaccines for children are less effective than earlier whole cell formulations (v. n. 8), and that protection wanes substantially after the last dose (v. n. 9).

(6) To check cross referencing we used a previously published systematic review. Using ${ }^{3}$ extracted numbers of true and false positive and negative results according to the reference

1 Cherry (2005: 1422-1427); Sheridan, Ware, Grimwood e Lambert (2012: 1434-1435); Olin, Rasmussen, Gustafsson, Hallander e Heijbel (1997: 1569-1577); Klein, Bartlett, Fireman, RowhaniRahbar e Baxter (2013).

2 Klein, Bartlett, Rowhani-Rahbar, Fireman e Baxter (2012, 367: 1012-1019); Misegades, Winter, Harriman, Talarico, Messonnier, Clark et al. (2010-2012, 308: 2126-2132) (British Medical Journal, Jul 2013, p. 1).

3 Stein, Hull, Patel, Olson, Ghali, Brant et al. (2004: 589-602) (British Medical Journal, May 2013, p. 2). 
tests, we reconstructed $2 \times 2$ tables for the patients with a non-high clinical probability and stratified them by predefined age categories ( $\leq 50$ years, $51-60$ years, $61-70$ years, $71-80$ years, and $>80$ years).

L'esempio (5) presenta due casi di sintagmi verbali complessi vaghi. Varied dovrebbe definire le ragioni dell'aumento (Reasons for the increase), ma il verbo stesso presenta una semantica vaga (De Mauro 1982, Ballmer e Pinkal 1983, Voghera 1997), quindi non può chiarire e non chiarisce quali siano le ragioni. Varied è modificato da likely, anch'esso termine semanticamente vago. Successivamente, il sintagma verbale wanes è modificato da substantially, il quale non definisce con precisione i termini della decrescita.

Nell'esempio (6) published systematic review è modificato da previously, che dovrebbe definirne la data di pubblicazione, ma lo fa indicando un periodo di tempo vago: precedente rispetto alla pubblicazione dell'articolo sul British Medical Journal, ma non si sa di quanto e non si sa a quale pubblicazione si faccia riferimento. Il sintagma nominale complesso analizzato finora è reso ancora più vago dall'articolo indeterminativo $a$ che lo precede, rendendolo un sintagma nominale indefinito. In quest'esempio, come per il precedente esempio (1), si può parlare di cluster di markers.

\subsection{Incertezza e mitigazione nel British Medical Journal}

La vaghezza, mediante l'inserimento di bushes all'interno degli enunciati, è uno dei mezzi utilizzati per mitigare una proposizione. Nel parlato, vaghezza e incertezza sono spesso copresenti: la vaghezza è sintomo di incertezza da parte dell'autore, il quale, per deresponsabilizzarsi, ricorre all'uso di bushes. È sufficiente considerare frasi quali "Arriverò per le sette", "Saranno una decina di chilometri", "Costerà un paio di euro", per avere un'esemplificazione della vaghezza che veicola incertezza. Tuttavia, come abbiamo affermato in precedenza (cfr. par. 1.1), il RAs presenta diverse caratteristiche costitutive (Searle 2009), tra cui quella della precisione. Se nel testo medico-scientifico la vaghezza fosse sintomo di incertezza, come spesso lo è nel parlato e nella divulgazione scientifica, gli obiettivi degli articoli da noi analizzati risulterebbero contradditori dal momento che vorrebbero comunicare informazioni precise, ma lo farebbero in maniera vaga e incerta. Riprendendo in considerazione i diversi casi in cui, negli articoli scientifici del British Medical Journal analizzati nei paragrafi precedenti, gli autori hanno fatto ricorso alla vaghezza, risulta evidente che questa strategia di mitigazione è riscontrabile solo a livello mono-enunciativo: se, da una dimensione di analisi enunciativa, si passa a una dimensione di analisi testuale, che prende quindi in considerazione co-testo e paratesti, è possibile constatare che tutti gli elementi vaghi sono precisati. Infatti, in quasi tutte le occorrenze di bushes individuate nel nostro corpus, i termini vaghi sono poi specificati da note di tipo bibliografico (esempi (5) e (6)), parentesi (esempio (6)) e tabelle (esempio (1)). Per cui, nel genere specifico quale è il Ras, lo scopo della vaghezza non è veicolare incertezza, come spesso invece accade nel parlato e nei testi di divulgazione scientifica.

\subsection{Lo scopo della vaghezza nel British Medical Journal}

Come abbiamo visto nel paragrafo precedente, la presenza di vaghezza non è causata da incertezza da parte degli autori degli articoli. Dunque, ci siamo chiesti quale sia lo scopo 
della vaghezza nel British Medical Journal. Tra le caratteristiche del RAs abbiamo anche indicato quella della chiarezza espositiva (cfr. par. 1.1). Ebbene, il ricorso alla vaghezza a livello enunciativo negli articoli del British Medical Journal sembra trovare motivazione in questioni meramente stilistiche, cioè nella volontà di rendere più scorrevole la lettura, senza appesantirla con dati numerici precisi. Questa ipotesi, trova conferma nelle istruzioni per gli autori, nelle quali si specifica: "We do want your piece to be easy to read but also want it to be as scientifically accurate as possible" (Advice on writing and submitting original research articles, http:/www.British Medical Journal.com/about-British Medical Journal/ resources-authors/article-types/research).

Nei rari casi in cui i termini approssimati dai bushes non vengono precisati dai paratesti, è probabile che prevalga la volontà di garantire l'anonimato dei pazienti, anch'esso requisito richiesto esplicitamente nelle istruzioni per gli autori.

\section{HEDGES NEL BRITISH MEDICAL JOURNAL}

Gli hedges (Caffi 2001) sono la seconda tra le strategie di mitigazione più usate, dopo i bushes, all'interno del corpus da noi qui presentato.

Più di trent'anni di ricerca hanno fatto del termine hedge un lessema molto inflazionato, una definizione ad ombrello, sotto la quale si raggruppano di volta in volta markers con diverse funzioni che agiscono su livelli diversi dell'atto linguistico. Prince et alii (1982) vi raggruppano fenomeni mitiganti sia il contenuto proposizionale che forme di modali, più propriamente agenti sulla forza illocutoria dell'atto; Lyons (1977) è stato il primo a parlare di modalità e modalizzazione epistemica, intuizione che ha avuto enorme seguito (ricordiamo qui le due riletture di Coates e Leech 1980 e Venier 1991). Della relazione tra modalità epistemica e forza del performativo hanno parlato per primi G. Lakoff (1972, riletto poi anche da Overstreet 2011) e Fraser (1999). Per quanto riguarda il testo scientifico, Hyland (1998) raggruppa sotto questa categoria la totalità dei fenomeni osservati nella sua analisi, alcuni dei quali in Caffi (2001) rientrano tra i bushes o tra gli shields.

Secondo Caffi (2001), attraverso gli hedges si agisce sulla forza illocutoria dell'atto linguistico, introducendovi indirettezza.

Dopo aver analizzato il corpus di articoli del British Medical Journal, abbiamo scelto di ordinare questa categoria di mitigatori in tre sottocategorie (cfr. Figura 2).

Abbiamo chiamato il primo tipo di siepi, che agiscono sulle condizioni A austiniane, relative al diritto di richiamare una competenza o un'autorità, siepi relative all'autore, in quanto la loro azione è focalizzata sul proteggere le dichiarazioni di chi scrive, solitamente con dichiarazioni preventive di tipo cautelativo (disclaimers).

Il secondo tipo di strategia, che abbiamo chiamato siepi relative al messaggio, coinvolge la condizione preparatoria B enunciata da Austin (2001), che prevede lo svolgimento corretto e completo di una procedura, segnalando approssimazione nella formulazione dell'atto linguistico (Caffi 2001).

L'obiettivo di questo tipo di strategia è quello di introdurre incertezza nell'enunciazione dell'atto linguistico, in modo da smorzare la forza della dichiarazione ed evitare critiche severe; se con la prima strategia gli autori adoperavano falsa modestia, rinunciando all'autorità attribuita loro dalla posizione di esperti, qui l'oggetto dell'azione mitigante è il messaggio, che arriva al lettore approssimato. 
L'ultima sotto-categoria, che chiamiamo siepi relative al contenuto, prevede l'impiego di mitigatori per affievolire l'impegno del parlante verso ciò che dice ed in particolare, viene messa in discussione la condizione essenziale per gli atti assertivi, cioè che la proposizione rappresenta uno stato di cose (Caffi 2001). Gli autori utilizzano strategie, quali i verbi immaginare, dire, a volte la proposizione condizionale oppure forme che rendono evidente che ci si sta muovendo nel campo della probabilità, come gli avverbi probabilmente e possibilmente.

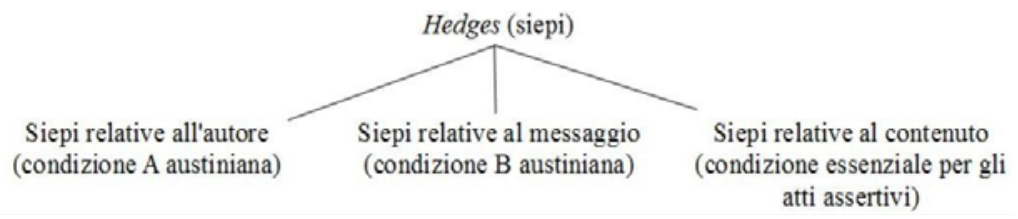

Figura 2. Hedges (siepi).

Come detto sopra (cfr. par. 1.1), un approccio al testo scientifico che guardi alla mitigazione rappresenta una vera e propria sfida: l'empiricità del modello unita alle specificità del genere scientifico hanno determinato un riadattamento sostanziale del modo di vedere l'agire delle tre strategie. Non tutte le citazioni sono schermi come non tutti i modali sono markers di mitigazione: le analisi dei corpora unite a lunghe riflessioni hanno portato allo schema seguente (fig. 3), che riassume la classificazione da noi proposta per gli hedges all'interno del corpus di ricerca del British Medical Journal.

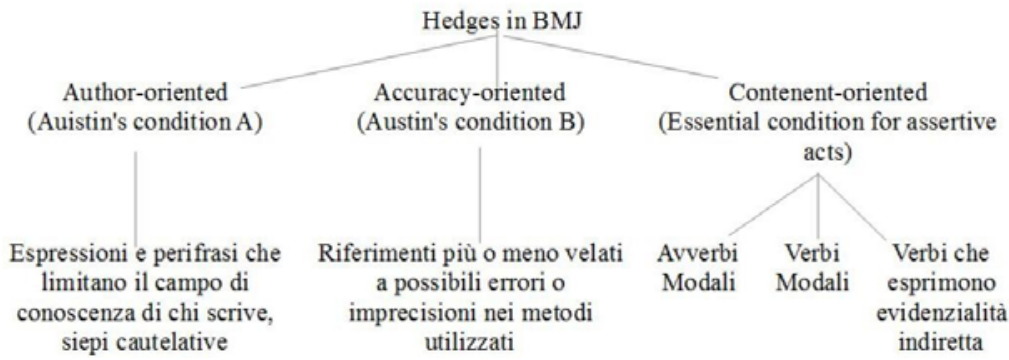

Figura 3. Hedges in BMJ.

\subsection{Author-oriented hedges}

In questa categoria rientrano tutti quei fenomeni di siepe cautelativa o disclaimers (Caffi 2001), che trovano la loro traduzione sul piano retorico con la mossa del mettere le mani avanti e che già Hyland (1998) elenca fra i writer-oriented hedges.

Il genere scientifico prevede vincoli strettissimi per quanto riguarda competenze e conoscenze degli autori: nell'opera a setaccio (Bastide 2001), lo scienziato deve tirare fuori dall'indifferenziato i risultati delle sue analisi, vedendoli con assoluta certezza, senza nessun filtro. Nel nostro caso, denunciare in modo velato mancanze nelle conoscenze, oppure nelle possibilità di intervento, limita la possibilità di critica verso le dichiarazioni appena fatte (cfr. esempio (7)). 
(7) Strategies to decrease the incidence of pertussis should prioritize giving the Tdap booster to people who received only acellular pertussis vaccines as children, as California has done since the 2010 outbreak, requiring Tdap vaccination for middle school students. To our knowledge this is the first study to assess the effectiveness of the Tdap booster in members of this new generation that has received all acellular vaccines. We had limited power to assess the effectiveness of Tdap vaccines in the subgroup of people born before 1950 (British Medical Journal, Jul 2013: 10)

Nell'esempio, ci sembra utile notare innanzitutto l'uso indiretto di should, che agisce da commissivo mascherando l'azione esercitiva. Molto spesso l'azione della modalizzazione e delle siepi non tende tanto a coprire vere e proprie lacune, quanto ad agire come mossa retorica nel gioco strategico delle critiche velate ad altri lavori e l'esercitivo prevede, per essere compiutamente felice, il manifestarsi di una posizione di comando, di subalternità. Veicola qui, quindi, una sorta di selfie autoriale, nel quale gli autori stessi, proprio mitigandone la portata esplicita, fanno passare un'idea di giustificata asimmetria. Ancora da notare le due siepi author-oriented, To our knowledge e We had limited power, dove è evidente l'intenzione cautelativa, rafforzata nel secondo caso dallo shift verso la forma più impegnata e minacciante la faccia (Goffman 1978) del we.

\subsection{Accuracy-oriented hedges}

Questa categoria vuole evidenziare quelle siepi che segnalano una mancata accuratezza nelle procedure di selezione dei dati; nel nostro caso, si tratta di un rimandare a possibili deficit strutturali nei sistemi di calcolo digitale o nei modelli statistici utilizzati. Talvolta, possono essere anche delle velate critiche ai corpora di dati utilizzati. Proseguendo con la metafora di Bastide (2001; cfr. supra), attraverso questi markers gli autori attribuiscono possibili errori od imprecisioni nei risultati ad un difetto strutturale del setaccio, per eccesso (maglie troppo larghe) o per difetto (troppo strette).

Nonostante la sinonimia, quindi, questa categoria non ha nulla a che vedere con quella proposta da Hyland (1998), dove venivano raggruppati quelli da lui chiamati propositional hedges, alcuni dei quali presenti tra i bushes da noi individuati (cfr. par. 2.1)

(8) Diabetes and cancer are routine diagnoses, and information on their occurrence has probably been collected in a large number of observational datasets, perhaps in the range of hundreds. If one were to also include hospital records, there are probably thousands of datasets that could generate estimates of associations between type 2 diabetes and incidence and mortality for each cancer. Only a fraction of them are probably assessed, a smaller fraction is written up for publication, and an even smaller fraction is published, after peer review that is filtered by reviewers who might have specific beliefs about the presence of these associations. It is possible that only a small proportion of the potentially available data are eventually included in published analyses. Ideally, one hopes that peer review has selected the best studies and the best data for publication. It is also possible, however, that bias in this literature could manifest itself as confirmation/allegiance bias. If there is strong belief in the specialty about the association between type 2 diabetes and risk of developing cancer, research efforts are under pressure to conform to this hypothesis when and if they are to be published (British Medical Journal, Jan 2015: 9) 
L'esempio (8) invece mostra, oltre ad una commistione forte fra cespugli e siepi, un uso particolare di accuracy-oriented hedge, molto vicina agli schermi di fizionalizzazione, quelli cioè che aprono ad un mondo possibile (Caffi 2001). "Ideally" introduce una velata ma non troppo implicita critica al sistema editoriale delle riviste scientifiche, scaricando su un'allusa scarsa professionalità, eventuali bias della ricerca all'oggetto dell'articolo, perché semplicemente ereditati. La critica viene qui rafforzata dal commissivo "to hope", che qui maschera un esercitivo.

\subsection{Content-oriented hedges}

Questa è la categoria di hedges che ricorre con più frequenza nel nostro corpus (nel 75\% dei casi). Sono le siepi prototipiche, che, attraverso l'uso strategico della modalizzazione, riducono il commitment epistemico verso le dichiarazioni degli autori.

Nel testo scientifico, la certezza è il valore a cui bisogna tendere in fase di sperimentazione ed arrivare, senza dubbi, in fase di resoconto dei risultati. Interessante, come vedremo (cfr. par. 3.5), l'uso dialogico dei verbi evidenziali: il valore della certezza assoluta si esprime al meglio nell'identità, fondante il paradigma conoscitivo, tipico della scienza e poi passato alla conoscenza generale, sapere = vedere bene (Latour e Woolgar 1979; Latour 1998, 2000; Bastide 2001), che struttura la conoscenza attorno alla vista come organo percettivo privilegiato della verità. Lo scienziato, nella sua azione che procede dall'indifferenziato al nitido, fonda tutta la sua coerenza sulle fonti dirette di conoscenza, che evitano interferenze interpretative e fallacie doxastiche, prima fra tutte la fonte percettiva della vista. Eppure, nel corpus da noi analizzato e qui presentato, non sempre l'uso di verbi che esprimono evidenzialità diretta è fortemente sconsigliato e minoritario, probabilmente perché considerati troppo vincolanti verso il commitment epistemico. La certezza viene quindi affidata agli evidenziali indiretti (Chafe e Nichols 1986, Willet 1988, Plungian 2001, Aikhenvald 2004, Marin-Arrese 2004), costringendo gli autori a rendere più raffinato il loro utilizzo all'interno delle mosse di deresponsabilizzazione: molte volte, quindi, anche un evidenziale, che esprima tipicamente un'opinione o un risultato indotto e non dedotto empiricamente, può veicolare certezza.

Sulla scia di Lyons (1977) e delle riflessioni di G. Lakoff (1972), Fraser (1975) e Coates e Leech (1980), si può affermare che l'azione dei modali sulla forza illocutoria di un atto è ormai prototipica. Anche in questo caso, però, l'attenzione non deve mai spostarsi dalle tipicità del genere in analisi (quello dell'articolo di ricerca scientifica): la marcatezza di questi dispositivi risulta laddove le eventuali approssimazioni non siano dovute a calcoli di probabilità statistica, sempre e comunque specificati nelle tabelle allegate agli articoli. L'uso di markers, quali possibly e probably, sono stati evidenziati solo quando esprimano la necessità, da parte degli autori, di tutelare la loro faccia (Goffman 1978) o il loro lavoro e non siano in nessun caso specificati altrove.

(9) However, alternative mechanisms should be considered because lithium is not as potent in acute phase therapy as other antidepressants, which, in turn, do not seem to have similar antisuicidal efficacy. The antisuicidal effect estimated here is larger than the effect on mood episodes, raising the possibility of a specific effect. Possible mechanisms include an effect on aggression or impulsivity, both of which are associated with an increased 
risk of suicide. Lithium may decrease aggression and possibly impulsivity, which might mediate its antisuicidal effect. Similarly, several genes have been found to be associated with suicidal behaviour and abnormalities in the serotonin system in suicide attempters and completers have suggested a biological basis for suicidal behaviour. Understanding the mechanism by which lithium acts to decrease suicidal behaviour could lead to a better understanding of the neurobiology of suicide (British Medical Journal, Jun 2013: 12)

Nell'esempio (9), infine, oltre ad un ulteriore uso indiretto di should come commissivo, a mascherare però un esercitivo, possiamo notare il cluster di modali, usati per alleggerire il carico epistemico sull'autore.

\subsection{Evidenzialità e mitigazione nel British Medical Journal: un'introduzione}

Secondo le definizioni classiche, nel dominio dell'evidenzialità rientrano tutti quei markers che contribuiscono ad indicare la valutazione di chi scrive/legge verso la validità dell'informazione, data attraverso l'esplicitazione della fonte dalla quale l'informazione arriva (Chafe 1986, Willet 1988, Plungian 2001, Marin-Arrese 2007).

Seguendo Willet (1988), che fu il primo a notarlo, all'interno del macro-dominio dell'evidenzialità, si possono certamente distinguere due sotto-domini: evidenzialità diretta (ovvero informazioni esplicitamente ricavate da fonti dirette, percettive, prima fra tutte la vista) ed evidenzialità indiretta (suddivisa in informazioni riportate ed inferite). Successivamente Aikhenvald (2004, Figura 4), ha analizzato il rapporto che ciascuno dei due domini stringe con i parametri di certezza/incertezza: in un'ipotetica scala che vada dal dato percepito come maggiormente certo a quello percepito come maggiormente incerto, i vettori partirebbero dagli evidenziali diretti verso quelli indiretti.

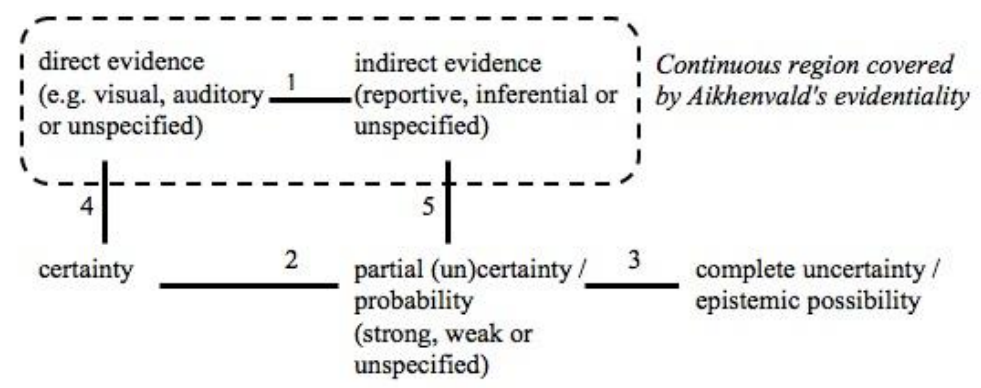

Figura 4. Evidenzialità (Aikhenvald 2004).

Come abbiamo già ricordato (cfr. par. 1.1), il metodo scientifico obbliga alla massima oggettività ed empiricità possibili, vincolando l'intero sistema di conoscenze della disciplina all'identità visto $=$ vero . L'articolo scientifico, quale particolare espressione del genere strutturalmente ben definito che è la scrittura scientifica, deve tendere a perdere qualsiasi impronta narrativa per diventare un perfetto resoconto dei processi avvenuti in laboratorio: chiaro, oggettivo, ripetibile.

Suggest può dunque essere riconosciuto come una siepe (Caffi 2001), in particolare come una siepe relativa al messaggio. In questa ipotesi, l'uso di suggerire rispetto ad osser- 
vare, può essere letto come una strategia atta a proteggere le dichiarazioni dell'autore che non si sbilancia troppo. La mitigazione/attenuazione agisce attraverso il marker evidenziale che codifica una valutazione minore dell'autore sulla validità della propria informazione (Auwera e Plungian 1988, Plungian 2001, Marín-Arrese 2007, Cornille e Marín-Arrese 2015).

A queste condizioni, ci aspettavamo una sovrabbondanza di evidenziali che esprimessero una conoscenza oggettiva e data da esperienze percettive dirette; al contrario, non solo gli evidenziali indiretti superano proporzionalmente, 4 a 1, gli altri, ma il parametro della certezza viene spesso veicolato proprio da questi ultimi. Inoltre, le sezioni assegnate dalla retorica del testo scientifico (Gross 1985, Swales 1990) ai dettagli ed all'esplicitazione dei risultati, luogo adibito per definizione alla certezza epistemica, sono le sezioni in cui le siepi relative al messaggio compaiono meno (10\%). La maggior parte degli evidenziali, il 77,7 \%, compare nelle sezioni introduzione e discussione.

Rimandiamo ad ulteriori approfondimenti e pubblicazioni future gli sviluppi di alcune ipotesi, come la connessione di questo particolare uso degli evidenziali con il grado 0 della scrittura scientifica (cfr. par. 4), il loro valore nel gioco retorico del testo scientifico ed una possibile gerarchizzazione di questi fenomeni in una scala che coinvolga anche il piano emotivo, secondo un maggiore/minore coinvolgimento del parametro evidentiality (Caffi e Janney 1994).

Ci limitiamo qui a qualche esempio che mostri come, nell'utilizzare l'evidenzialità come siepe, gli autori marchino le loro scelte instaurando un dialogo tra situazioni rette da verbi di inferenza, evidenziate perché immediatamente contrapposte a verbi di evidenzialità diretta. In particolare, di seguito si osservi il rapporto fra suggest ed observe.

(10) Although modulation of the glucagon-like peptide-1 system has been shown to have pleiotropic effects on the cardiovascular system, we did not observe any significant benefits, or risk, at the population level. However, our analyses also suggest that sitagliptin was prescribed in our cohort for patients with more advanced diabetes, given the higher rates of complications of diabetes at baseline and higher glycated hemoglobin values» (British Medical Journal, Ap. 2013: 4)

(11) Several pooled safety analyses have suggested potential benefits associated with dipeptidyl peptidase-4 inhibitors. These observational data provide evidence of the comparative safety of sitagliptin and support current recommendations to use sitagliptin as add-on treatment if needed in people with diabetes» (British Medical Journal, Ap. 2015: 6)

Nel primo esempio (10), entrambi i verbi veicolano certezza, rafforzati anche dall'uso del verbo to show; ma nel secondo esempio (11), il modale potential riesce a rafforzare l'uso di suggested come evidenziale indiretto, mettendolo in chiara contrapposizione con observe.

\subsection{Scopo dell'indirettezza nel British Medical Journal}

Le siepi, con la loro azione sulla forza illocutoria, assurgono solitamente alla funzione di deviare possibili critiche, rinunciando a posizioni forti verso le proprie dichiarazioni e diminuendo il proprio impegno epistemico verso le stesse.

Il loro utilizzo sembra giustificato alla luce di quelle che potrebbero essere i due vincoli (Latour e Woolgar 1979, Hyland 1998) della scrittura scientifica, le due principali tensioni 
alle quali è sottoposto chi si misura con questo genere: self-promotion, intesa come costruzione di una propria faccia (Goffman 1971) e promozione dell'originalità del proprio lavoro; modestia, intesa come inserimento cosciente in un filone letterario, che è la letteratura scientifica, guida di coloro che leggeranno e valuteranno il lavoro. Originalità da una parte, tradizione dall'altra: strategicamente, per affrontare i bisogni identitari, occorre cautela. La caution è dunque la cifra del bisogno di questo genere di mitigazione e, forse, dell'utilizzo della mitigazione tutta all'interno di questo genere testuale altrimenti perfettamente asettico.

\section{CONCLUSIONI}

In questo contributo abbiamo presentato parte dei risultati ottenuti nel tentativo di applicare il modello della mitigazione elaborato da Claudia Caffi (2001) al testo scritto medico-scientifico.

Innanzitutto possiamo affermare che in tutti gli articoli del corpus abbiamo riscontrato la presenza di tutti i due tipi di mitigatori su cui ci siamo concentrati (bushes e hedges).

Il modello Caffi pone le sue basi sull'identità forte tra mitigazione e deresponsabilizzazione, cioè la mitigazione è una forma e un mezzo di deresponsabilizzazione enunciativa. Questo, spesso, implica l'azione dei markers sul parametro dell'incertezza. Nel genere specifico e particolare del RAs, benché vi sia mitigazione, essa non corrisponde a deresponsabilizzazione e a incertezza da parte degli autori; al contrario, è frequente che i markers di mitigazione agiscano sul parametro opposto all'incertezza (cespugli; evidenziali che esprimono conoscenza inferita sulla base di risultati certi, "the evidence suggest"). Tuttavia, il modello rimane valido, anzi trova applicazione anche in un contesto lontano da quello per cui è stato definito, infatti le sue regole regolative (Searle 2009) trovano conferma: è frequente l'azione congiunta di più mitigatori (cluster di mitigatori); si verifica co-varianza di mitigatori (un mitigatore o un cluster di mitigatori interviene su più parametri) co-orientata e non co-orientata; per comprendere tutte le funzioni dei markers e la loro capacità d'intervenire su più parametri, è necessario attuare il passaggio da un'analisi esclusivamente mono-enunciativa a un'analisi pluri-enunciativa, cioè dal livello frasale al livello testuale. Riteniamo inoltre che, all'interno di un'analisi svolta sul piano testuale, si renda evidente la necessità di definire e, solo all'interno di un'analisi testuale, si possa riuscire a definire un grado zero proprio del genere, che ci permetta di distinguere ciò che è marcato da ciò che non lo è.

Le nostre ricerche future saranno volte a tentare di definire quello che è il grado zero del genere medico-scientifico: cosa cioè caratterizzi questo genere testuale, quale sia la sua quidditas. In relazione alla mitigazione, quale sia la base sulla quale essa agisce e cosa rimanga, una volta isolati i fenomeni mitigatori, sul piano illocutorio; verso il perlocutorio, se esista, e di quale natura sia, una relazione tra mitigazione e permeabilità del messaggio scientifico.

\section{Riferimenti bibliografici}

Adolphs, S., Atkins, S. e Harvey, K. (2007). Caught Between Professional Requirements and Interpersonal Needs: Vague Language in Healthcare Contexts. In Cutting, J. (ed.). Vague language explored. London: Palgrave Mcmillan, pp. 62-78.

Aïkhenvald, A. (2004). Evidentiality. Oxford: Oxford University Press.

Austin, J. L. (2011 [1962]). Come fare cose con le parole (trad. C. Villata). Genova: Marietti. 
Auwera, V. e V. Plungian (2006). "Towards a typology of discontinuous past marking”, Sprachtypol. Univ. Forsch (STUF), Berlin, 4, pp. 317-349.

Ballmer, T. e M. Pinkal (1983). "Introduction”. In Ballmer, T. e M. Pinkal (eds.). Approaching vagueness. Amsterdam: North-Holland, pp. 1-10.

Bastide, F. (2001). Una notte con Saturno. Scritti semiotici sul discorso scientifico. Roma: Meltemi.

Bauman, Z. (2003). Modernità liquida. Roma-Bari: Laterza.

Bazerman, C. (1988). Shaping Written Knowledge: The Genre and Activity of Experimental Article in Science. Madison: University of Wisconsin Press.

Bazerman, C. (1991 [1988]). Le origini della scrittura scientifica: come è nata e come funziona l'argomentazione del saggio sperimentale. Ancona: Transeuropa.

Bazzanella, C. (2011). Numeri per parlare. Da "quattro chiacchiere" a "grazie mille". Roma-Bari: Laterza.

Beck, U. (2000). La società del rischio. Verso una seconda modernità. Milano: Carocci.

Bühler, K. (1983 [1934]). Teoria del linguaggio. Roma: Editore Armando.

Caffi, C. (1994). Metapragmatics. In Asher, R. and J. Simpson (eds.). The Encyclopedia of Language and Linguistics. Oxford: Pergamon.

Caffi, C. (2001). La mitigazione. Un approccio pragmatico alla comunicazione nei contesti terapeutici. Münster: LIT.

Caffi, C. (2017). "La mitigazione: tappe di un itinerario di ricerca", Normas, 7, pp. 4-18.

Caffi, C., Domaneschi, F., Marinai, M., Stancampiano, D., Ierardi, S. and Bonelli, L. (2016). Mitigating devices and strategies in the "British Medical Journal". Paper presented at the Workshop on Certainty and Uncertainty in Biomedical Scientific Communication, University of Genoa, 27 April 2016.

Caffi, C. e R. Janney (1994). Toward a pragmatics of emotive communication. In Caffi, C. e R., Janney (eds.). Involvement in language, special issue of Journal of Pragmatics, 22, Nos. 3/4, pp. 325-373.

Carter, R. e M. McCarthy (2006). Cambridge Grammar of English. Cambridge: Cambridge University Press.

Castellas, M. (2012), La nascita della società in rete, Milano, UBE.

Chafe, W. (1986). "Evidentiality in English Conversation and Academic Writing”. In W. L. Chafe, \& J. Nichols (Eds.), Evidentiality: The Linguistic Coding of Epistemology. Norwood, NJ: Ablex, pp. 261-272.

Chafe, W. e J. Nichols (ed.) (1986). Evidentiality: The Linguistic Coding of Epistemology. Norwood NJ: Ablex Publishing Corpotation.

Channell, J. (1994). Vague language. Oxford: Oxford University Press.

Cherry, J.D. (2005): "The epidemiology of pertussis: a comparison of the epidemiology of the disease pertussis with the epidemiology of Bordetella pertussis infection", Pediatrics, 115 (5): 1422-1427.

Coates, J. e G. Leech (1980). "The Meaning of the Modals in British and American English", York Papers in Linguistic, 8, pp. 23-34.

Cornille, B. e J. Marin Arrese (2015). "Evidentiality and the Semantics Pragmatics Interface", Journal of Linguistics, 29.

De Mauro, T. (1982). Minisemantica dei linguaggi non verbali e delle lingue. Roma-Bari: Laterza.

Feyerabend, P. (2013 [1975]). Contro il metodo. Abbozzo di una teoria anarchica della conoscenza. Milano: Feltrinelli.

Fraser, B. (1999). "What are discourse markers", Journal of Pragmatics, 31, 7, pp. 931-951.

Gallardo, S. (2005), "Pragmatic support of medical recommendations in popularized texts", Journal of Pragmatics, 37, 6, pp. 813-835.

Goffman, E. (1971 [1967]). Il rituale dell'interazione. Bologna: Il Mulino.

Goffman, E. (1987 [1981]). Forme del parlare. Bologna: Il Mulino.

Grabe, W. e R. Kaplan (1997). "On Writing of Science and the Science of Writing: Hedging in Science text and Elsewhere". In Markkanen, R. and H. Schröder (eds.). Hedging in Discourse. Approaches to the Analysis of a pragmatic Phenomenon in Academic Texts. Berlino: de Gruyter, pp. 151-167. 
Gross, A. (1985). "The form of the experimental paper: A realisation of the myth of induction", Journal of Technical Writing and communication, 15, pp. 15-26.

Halliday, M. (2004a). "The Language of Science". In Webster, J.J. (ed.). The Collected Works of M.A.K. Halliday. London-New York: Continuum International Publishing, vol. 5.

Halliday, M. (2004b), "Language and Education". In Webster, J.J. (ed.). The Collected Works of M.A.K. Halliday. London-New York: Continuum International Publishing, vol. 9.

Halliday, M. e J. Martin (1993). Writing Science: Literacy and Discourse Power. Pittsburgh: University Press of Pittsburgh.

Hyland, K. (1998). Hedging in scientific research articles. Amsterdam-Philadelphia: John Benjamins Publishing Company.

Hyland, K. (2002). "Authority and invisibility: authorial identity in academic writing", Journal of Pragmatics, 34, 8, pp. 1091-1110.

Hyland, K. (2009). Academic Discourse. London-New York: Continuum International Publishing.

Hyland, K. e C. Guinda Sancho (2012). Stance and Voice in Written Academic Genres. London: Palgrave Macmillan.

Klein, N., Bartlett, J., Fireman, B., Rowhani-Rahbar, A. e R. Baxter (2013): "Comparative effectiveness of acellular versus whole cell pertussis vaccines in teenagers", Pediatrics, 131 (6): 1717-1722.

Klein, N. P., Bartlett, J., Rowhani-Rahbar, A., Fireman, B. e R. Baxter (2012): "Waning protection after fifth dose of acellular pertussis vaccine in children", $N$ Engl J Med, 367: 1012-1019.

Knorr-Cetina, K. (1999). Epistemic cultures. How the Science make Knowledge. Harvard: Harvard University Press.

Knorr-Cetina, K. (2007). "Culture in global knowledge societies: knowledge culture and epistemic culture”. In Interdisciplinary Science Rewiews, 32, 4, pp. 361-375.

Lakoff, G. (1973 [1972]). "Hedges: A study in meaning criteria and the logic of fuzzy concepts", Journal of Philosophical Logic, 2, pp. 458-508.

Latour, B. e S. Woolgar (1979). Laboratory life: the social construction of scientific facts. London: Sage.

Latour, B. (1998 [1989]). La scienza in azione. Introduzione alla sociologia della scienza. Torino: Edizioni di comunità..

Latour, B. (2000). Pandora's Hope: Essays on the Reality of Science Studies. Harvard: Harvard University Press.

Lévi-Strauss, C. (1964 [1962]). Il totemismo oggi. Milano: Feltrinelli.

Mangani, G. (1991). Una retorica del testo scientifico. In Bazerman, C. (ed.). Le origini della scrittura scientifica: come è nata e come funziona l'argomentazione del saggio sperimentale. Ancona: Transeuropa.

Marin Arrese, J. (2007). "Commitment and Subjectivity in the Discourse of Opinion columns and Leading articles", RAEL, 1.

Merton, R. (1968). Social Theory and Social Structure. New York: The Free Press.

Merton, R. (1981 [1973]). La Sociologia della Scienza. Indagini Teoriche ed Empiriche. Milano: FrancoAngeli.

Meyer, P. G. (1997). "Hedging strategies in written academic discourse: strengthening the argument by weakening the claim”. In Markkanen, R. and H. Schröder (eds.), Hedging and discourse: approaches to the analysis of a pragmatic phenomenon in academic texts. Berlin: de Gruyter, pp. 21-41.

Mey, J. L. (in corso di pubblicazione). When hedging fails. Evidentials and (Un)certainty. Paper presentato nel Workshop on Certainty and Uncertainty in Biomedical Scientific Communication, Università di Genova, Italia, 27 aprile 2016.

Mihatsch, W. (2010). "The diachrony of rounders and adaptors: approximation and unidirectional change”. In Kaltenbök, G. et alii (2010). New approaches to hedging. Bingle y: Emerald, pp. 93-122. 
Misegades, L. K., Winter, K., Harriman. K., Talarico, J., Messonnier, N.E., Clark, T. A., et al. (2012): "Association of childhood pertussis with receipt of 5 doses of pertussis vaccine by time since last vaccine dose", California (2010), JAMA (2012), 308: 2126-2132 (British Medical Journal, Jul 2013, p. 1).

Olin, P., Rasmussen, F., Gustafsson, L., Hallander, H.O. e H. Heijbel (1997): "Randomised controlled trial of two-component, three-component, and five-component acellular pertussis vaccines compared with whole-cell pertussis vaccine. Ad Hoc Group for the Study of Pertussis Vaccines", Lancet, 350: 1569-1577.

Overstreet, M. (2011). "Vagueness and hedging". In Andersen, G. e K. Aijmer (eds.). Handbooks of Pragmatics: Pragmatics of Society. Berlino: Mouton de Gruyter, pp. 293-318.

Parret, H. (1984). "Regularities, rules and strategies". In Caffi, C. (ed.), pp. 569-592.

Plungian, V. (2001). "The place of evidentiality within the universal grammatical space", Journal of Pragmatics, 33, pp. 349-357.

Polany, M. (1946). Science, faith and Society. London: Oxford University Press.

Polany, M. (1962 [1958]). Personal Knowledge. Towards a Post-Critical Philosophy. London: Routledge.

Prince, E. F., Frader, J. e C. Bosk (1982). "On hedging in Physician-Physician Discourse”. In Di Pietro, J. (ed.). Linguistics and the Professions. Norwood/New Jersey: Ablex, pp. 83-97.

Sbisà, M. (ed.) (1978). Gli atti linguistici. Milano: Feltrinelli.

Sbisà, M., (2001). "Illocutionary force and degrees of strength in language use", Journal of Pragmatics, 33, pp. 1791-1814.

Sbisà, M., (2009). Linguaggio, azione, interazione, Trieste: EUT.

Searle, J. (2009 [1969]). Atti linguistici. Saggio di filosofia del linguaggio. Torino: Bollati Boringhieri.

Searle, J. (1978a [1975]). "Per una tassonomia degli atti illocutori". In Gli Atti linguistici, Sbisà, M. (ed.), Milano Feltrinelli, pp. 168-198;

Searle, J. (1978b [1975]). “Atti linguistici indiretti”. In Sbisà, M. (ed.), pp. 252-280.

Sheridan, S.L., Ware, R.S., Grimwood, K. e S. B. Lambert (2012): "Unexpectedly limited durability of immunity following acellular pertussis vaccination in preadolescents in a North American outbreak", Clin Infect Dis, 55: 1434-1435.

Skelton, J. (1997). "How to Tell the Truth in "The British Medical Journal": Patterns of Judgement in the 19 and 20Centuries". In Markkanen, R. and H. Schröder, (eds.). Hedging in Discourse. Approaches to the Analysis of a pragmatic Phenomenon in Academic Texts. Berlino: De Gruyter, pp. 42-63.

Stein, P. D., Hull, R. D., Patel, K. C., Olson, R. E., Ghali, W. A., Brant, R. et al. (2004): "D-dimer for the exclusion of acute venous thrombosis and pulmonary embolism: a systematic review", Ann Intern Med, 140: 589-602. (British Medical Journal, May 2013, p. 2).

Swales, J. (1990). Genre Analysis: English in academic and research settings. Cambridge: Cambridge University Press.

Van Eemeren, F. e R. Grootendorst (1982). Speech Acts in Argumentative Discussions. Cinnaminson: Foris Publications.

Venier, F. (1991). La modalizzazione assertiva. Avverbi modali e verbi parentetici, Pavia: Franco Angeli.

Voghera, M. (1997). "Qualche osservazione su significati lessicali e significati grammaticali”. In Leoni, A., Gambarara, D., Gensini, S., Lo Piparo, F. e R. Simone (eds.). Ai limiti del linguaggio. Vaghezza, significato storia. Roma-Bari: Laterza, pp. 127-137.

Voghera, M. (2012). "Chitarre, violino, banjo e cose del genere". In Thornton, A.M. e M. Voghera (eds.). Per Tullio De Mauro. Roma: Aracne, pp. 429-460.

Willet, T (1988). "A cross-linguistic survey of grammaticisation of evidentiality", Studies in Languages, 12, pp. 51-97. 